\title{
An assessment study of absorption effect: LED vs tungsten halogen lamp for noninvasive glucose detection
}

\author{
Nur Ain Mohd Aziz*,†, Norhana Arsad*,*, P. Susthitha Menon*, \\ Sahbudin Shaari*, Zalhan Md Yusof ${ }^{\dagger}$ and Abdur Rehman Laili ${ }^{\dagger}$ \\ *Institute of Microengineering and Nanoelectronics (IMEN), \\ Dept. of Electrical, Electronic and System Engineering \\ Faculty of Engineering and Built Environment \\ The National University of Malaysia, Bangi, Selangor \\ ${ }^{\dagger}$ Department of Photonics, MIMOS Berhad, \\ 57000 Technology Park Malaysia, Kuala Lumpur, Malaysia \\ *norhana@eng.ukm.my
}

Received 9 June 2014

Accepted 13 October 2014

Published 19 November 2014

\begin{abstract}
Noninvasive glucose monitoring development is critical for diabetic patient continuous monitoring. However, almost all the available devices are invasive and painful. Noninvasive methods such as using spectroscopy have shown some good results. Unfortunately, the drawback was that the tungsten halogen lamps usage that is impractical if applied on human skin. This paper compared the light emitting diode (LED) to traditional tungsten halogen lamps as light source for glucose detection where the type of light source plays an important role in achieving a good spectrum quality. Glucose concentration measurement has been developed as part of noninvasive technique using optical spectroscopy. Small change and overlapping in tungsten halogen results need to replace it with a more convenient light source such as LED. Based on the result obtained, the performance of LED for absorbance spectrum gives a significantly different and is directly proportional to the glucose concentration. The result shows a linear trend and successfully detects lowest at 60 to $160 \mathrm{mg} / \mathrm{dL}$ glucose concentration.
\end{abstract}

Keywords: Tungsten halogen; LED; glucose; reflectance; absorbance; spectrometer.

\section{Introduction}

Diabetes is a disease prior to glucose concentration level fluctuation in human blood. A number of techniques and technologies have been introduced to detect the glucose level both optically and non-optically.
The quality of a light spectrum is measured using an optical device called a spectrometer. The four major system components in a spectrometer system are the light source, sample preparation, interferometer and infrared detector. When two light beams which

\$Corresponding author.

This is an Open Access article published by World Scientific Publishing Company. It is distributed under the terms of the Creative Commons Attribution 3.0 (CC-BY) License. Further distribution of this work is permitted, provided the original work is properly cited. 
have traveled different paths meet, this will produce an interference pattern. ${ }^{1}$ Light is an important component for collecting the spectrum using spectroscopy technique. Some basic properties of the sample can be determined by the wavelengths and amount of light absorbed by the sample at each wavelength.

Denoting A as absorbance value, $\varepsilon$ as absorptivity, $\iota$ as path length and $\mathrm{c}$ as concentration. The formula Beer-Lambert Law given in Eq. (1) is useful to determine the amount of light absorbed

$$
A=\varepsilon \iota c .
$$

Tungsten halogen lamp is most commonly used as the light source for the spectrometer setup. Most available devices for glucose monitoring require extracting of blood from diabetic or physical contact to human body. ${ }^{2,3}$ Many researchers have tried to develop a noninvasive glucose monitoring device by using light intensity and spectrum to measure human biological properties. ${ }^{4,5}$ This approach would provide a safer and a more convenient method to monitor and control diabetes. ${ }^{6,7}$ The in vitro measurement of glucose in human physiological fluids is of great importance in clinical diagnosis of metabolic disorders.

Different research groups around the world have been proposing near-infrared (NIR) glucose detection due to its higher penetration depth to the skin for quantitative analysis. ${ }^{2}$ Tungsten halogen interferometry is the active type of light source for interferometry used in making an absolute measurement. ${ }^{8}$ Light emitting diode (LED) can be used to improve and simplify the white light problem. ${ }^{9}$ Hence, a study on LED and tungsten halogen characteristic is carried out to test whether an alternative light source can do better and improve the performance. A study of LED in human skin has been proven that at wavelength 970 to $10,600 \mathrm{~nm}$ can penetrate into the dermis of skin layer. ${ }^{10}$

\section{Experimental Design}

An Arcoptic spectrometer with InGaAs photodiode detector was used in the setup. This setup was performed with the NIR spectrometer calibrated for measurements within the range of $900-2500 \mathrm{~nm}$. The NIR spectrometer came with software to display the spectral measurement result in real time. The software performs Fourier Transform to convert the recorded interferogram to a spectrogram. For reference measurement, a NIR spectrometer equipped with tungsten halogen as the light source providing 360-2500 $\mathrm{nm}$ spectral range was also prepared. This allows a comparison analysis with the LED. A possible method of analyzing and predicting glucose concentration was suggested between wavelengths 1100 and $2450 \mathrm{~nm} .{ }^{11}$ The selected LED with center wavelengths of $1200 \mathrm{~nm}$ was explored in this experiment to see their effect on the glucose sample. The LEDs were chosen based on the NIR range and availability in the market in the form where the diode is encapsulated in a round clear $5 \mathrm{~mm}$ diameter epoxy casing. Furthermore, there is also research at wavelength of 1100-1380 nm which showed the possibility of glucose detection. ${ }^{12}$ For the purpose of quantitative analysis, four levels of glucose concentrations were proposed to be tested in the experiment. 0.5 of $\alpha$-d-glucose powder from Sigma Aldrich was dissolved in $100 \mathrm{~mL}$ of deionized water to form $0.09 \mathrm{M}$ standard solution. $60-160 \mathrm{mg} /$ $\mathrm{dL}$ with an interval of $10 \mathrm{mg} / \mathrm{dL}$ of glucose level solutions were prepared by dilution process using a pipette. The glucose concentrations chosen have physiological relevance to the human body. This experiment was used to link the optical NIR utilizing LED to glucose concentration.

\subsection{Reflectance technique}

Reflectance relies on the focused projection of the spectrometer beam into the sample where it is reflected, scattered and transmitted through the sample. $1 \mathrm{~cm}$ path length is used for the setup. A special holder is designed to make sure the path length remains the same in all the experiments.

A bifurcated fiber probe is used as a waveguide in the experiment. The basic setup in using the bifurcated fiber probe is one of the bifurcated ends need to be connected to a light source, such as tungsten halogen light, while the other is connected to the NIR spectrometer and the sample. In this setup, the light will travel through the first bifurcated end to the distal end of the probe. The probe has one and six fiber configurations for source and reflection, respectively. The reflected light from the sample will then travel from the distal end to the second bifurcated end and into the spectrometer for analysis. This is illustrated in Fig. 1.

In exploring the LED light source in reflectance method, the LED configuration as shown in Fig. 2 has been proposed in order to make sure the light covered the cuvette surface. The setup also needs to 


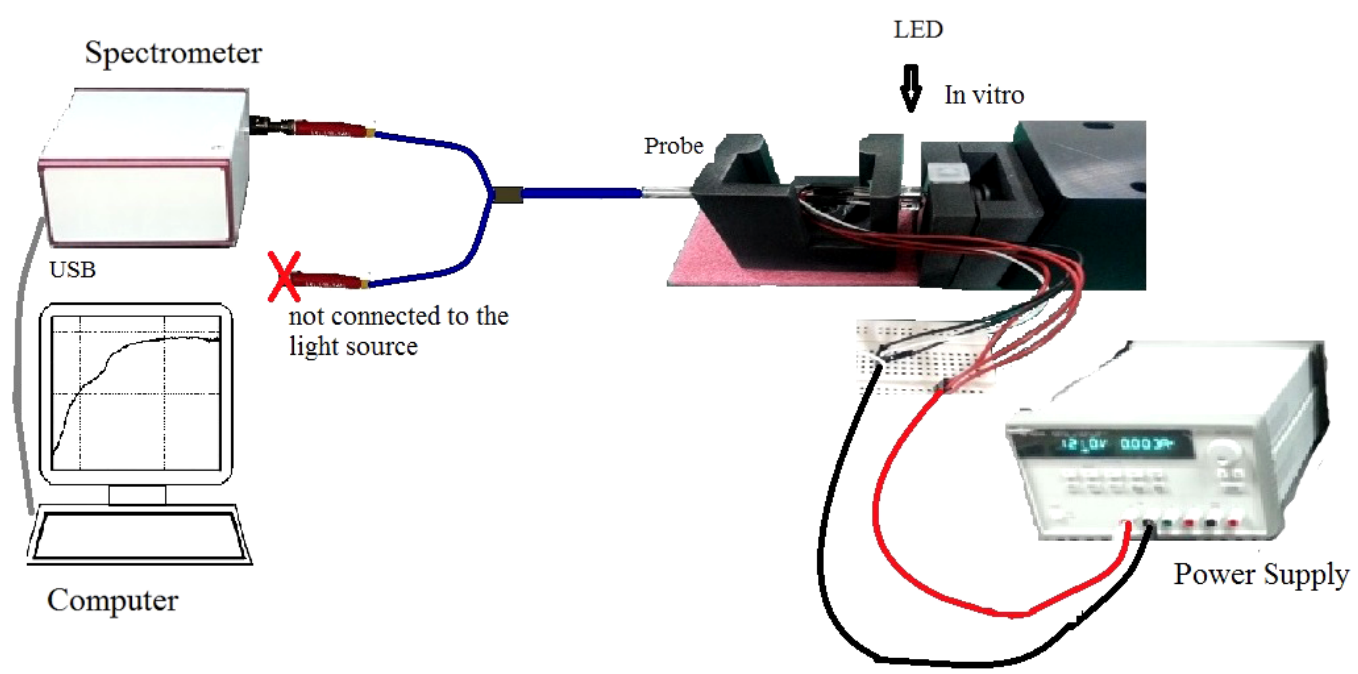

Fig. 1. Reflectance setup using LED as the light source.

be fixed this way in order to eliminate the background in this experiment. The LEDs have to be placed in a circle of diameter $1 \mathrm{~cm}$ to make sure all the light travels without being blocked by the holder itself. $1.32 \mathrm{~V}$ power is required to turn on the LED.

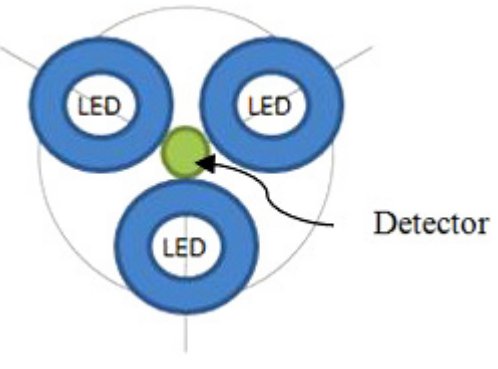

Fig. 2. LED configuration.

\section{Results and Discussion}

Figure 3 shows examples of NIR absorbance spectrum using a LED1200E with light reflected through the cuvette. The majority of the spectral variations observed in Fig. 3 are the result of glucose concentration variations. We obtained 11 readings of glucose in aqueous from 60 to $160 \mathrm{mg} /$ $\mathrm{dL}$ with an interval of $10 \mathrm{mg} / \mathrm{dL}$.

The InGaAs detector in the spectrometer was able to collect the spectrum from the LED1200E with a central wavelength of $1200 \mathrm{~nm}$. There are significant differences when glucose concentration was varied and this will help in data analysis by using the chemometric model.

Figures 4(a) and 4(b) are purposely magnified to visualize the increment of each absorbance spectrum. The result shows that the absorbance

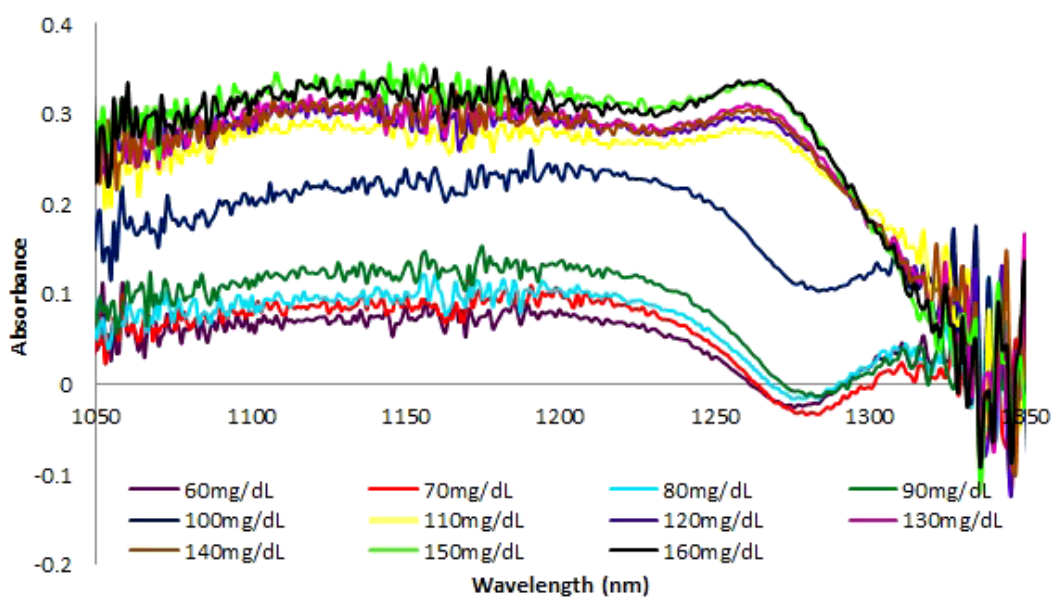

Fig. 3. Absorbance spectrum with various glucose concentrations. 
N. A. M. Aziz et al.

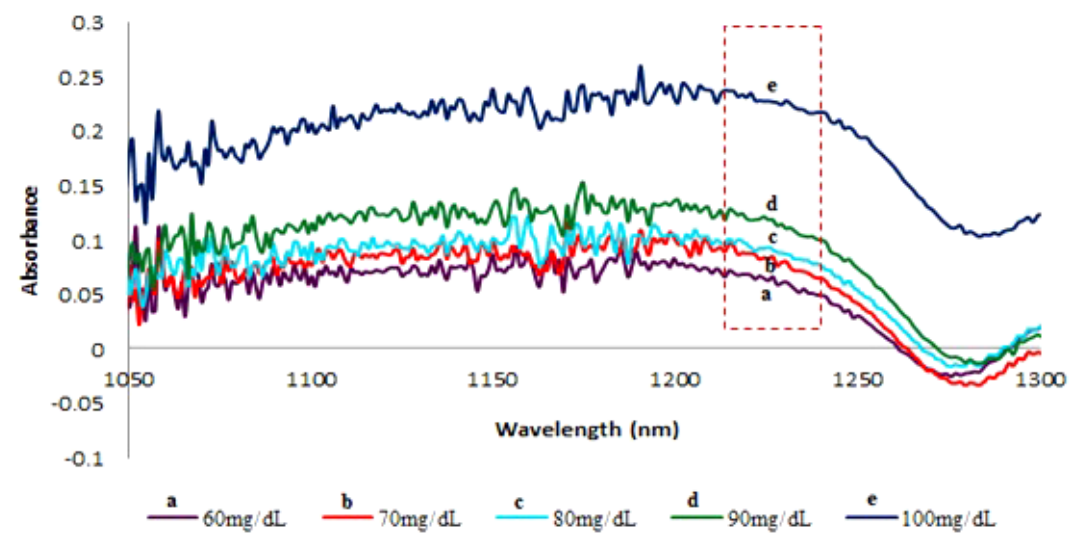

(a)

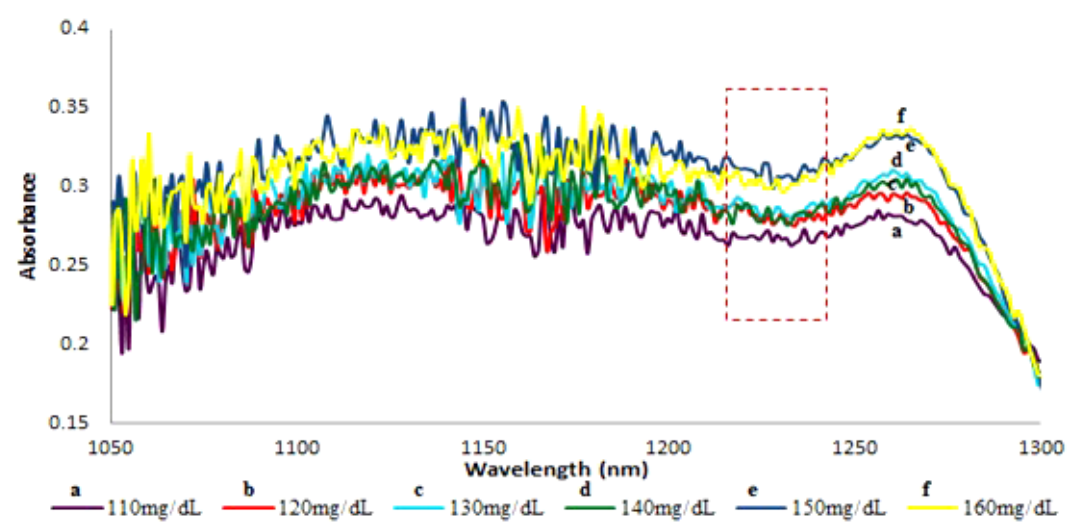

(b)

Fig. 4. Absorbance spectrum for LED1200E with (a) glucose concentration range of 60 to $100 \mathrm{mg} / \mathrm{dL}$ (b) glucose concentration range of 110 to $160 \mathrm{mg} / \mathrm{dL}$.

spectrum is directly proportional to the glucose concentration and thus implies the Beer-Lambert Law. It has been said that, the increment in the absorbance spectrum is due to the increased amount of light absorbed by the glucose molecule in the sample. Referring to the dotted region (wavelength $1226 \mathrm{~nm}$ ), the minimum absorbance spectrum is $0.07 \mathrm{AU}$ and the maximum absorbance spectrum is $0.31 \mathrm{AU}$.

The data collected using tungsten halogen as the light source are shown in Fig. 5. The overall absorbance spectrum generated produced overlapping output for different glucose concentrations. Referring to the dotted region (wavelength $1080 \mathrm{~nm}$ ), the minimum absorbance spectrum is $-0.015 \mathrm{AU}$ and the maximum absorbance spectrum is $0.016 \mathrm{AU}$.

The results obtained in Fig. 4 shows significantly different absorbance spectrum of glucose concentration but the results in Fig. 5 shows some overlapping and minimum change in absorbance spectrum. The obvious change in the absorbance spectrum is useful in chemometric analysis for glucose prediction.

Result from LED is undeniably excellent compared to tungsten halogen. This is due to difference in total power output of both light sources. Tungsten halogen absolute power at $1200 \mathrm{~nm}$ is $31.6 \mathrm{~mW}$ while combination of 3 LED $1200 \mathrm{~nm}$ produces $37.8 \mathrm{~mW}(12.6 \mathrm{~mW} / \mathrm{LED})$. Combination of LED source produces higher radiant power density thus higher amount of photon emitted to the sample.

The challenge in in vitro glucose detection is to detect low level of glucose concentration. In our case, the glucose concentration is $60-160 \mathrm{mg} / \mathrm{dL}$ with an interval of $10 \mathrm{mg} / \mathrm{dL}$. According to Burns an increment of $10 \mathrm{mg} / \mathrm{dL}$ of glucose concentration contribute only to an increment of less than $10 \mu \mathrm{AU}$ in the absorbance spectrum. ${ }^{13}$ The absorbance spectrum shown in Fig. 5 produced an overlapping output due to the minimal change in absorbance. In 


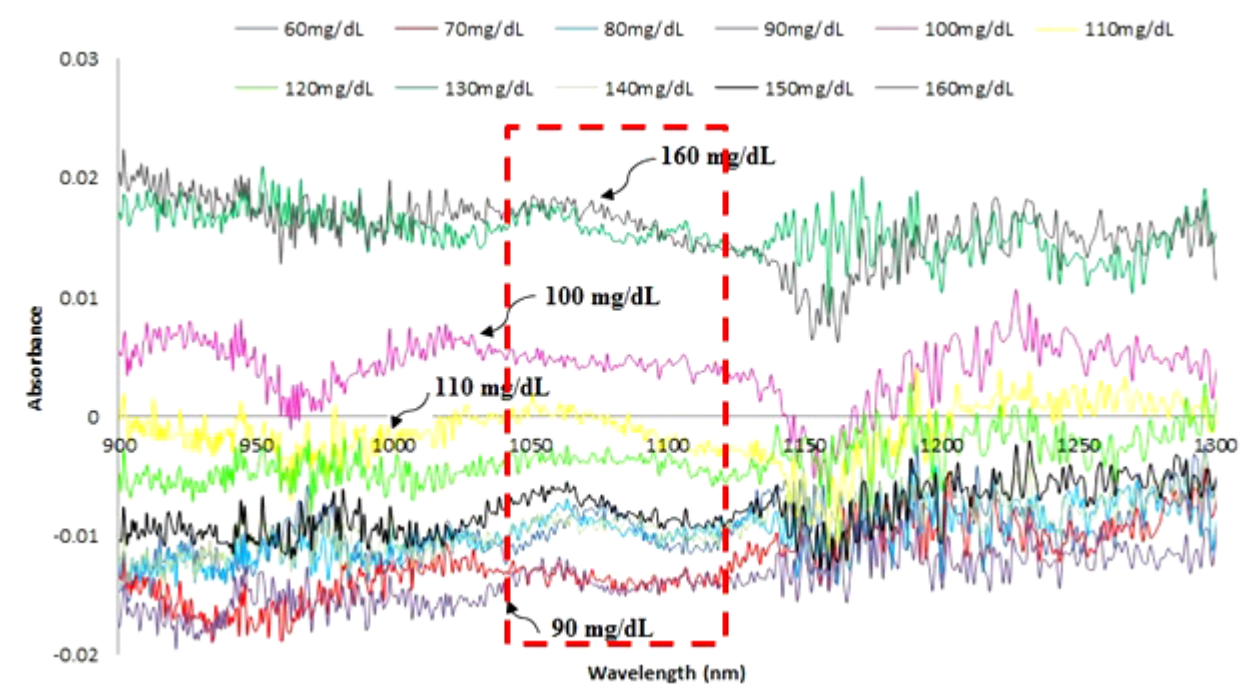

Fig. 5. Absorbance spectrum for tungsten halogen with glucose concentration range 60 to $160 \mathrm{mg} / \mathrm{dL}$.

order to overcome the overlapping spectrum produced by tungsten halogen, the LED was explored and found to give a good-quality spectrum. The maximum absorbance spectrum is $0.31 \mathrm{AU}$ and the minimum absorbance spectrum is 0.07 for LED1200E and proved the obvious change in the absorbance spectrum.

\section{Conclusion}

LED1200E is capable to measure low-range glucose concentration which is $60-160 \mathrm{mg} / \mathrm{dL}$. It is observed that LED1200E produces significantly different absorbance spectra when a difference of $10 \mathrm{mg} / \mathrm{dL}$ in glucose concentration is measured. Tungsten halogen can be used to collect the glucose spectrum in order to identify the specific wavelength that react to glucose vibration, but LED1200E is more convenient to use in the prediction of glucose level. Thus, LED can be considered as the better light source for glucose detection than tungsten halogen lamp due to the stability of its light intensity.

\section{References}

1. B. C. Smith, Fundamentals of Fourier Transform Infrared Spectroscopy, CRC Press, 2011.

2. R. Marbach et al., "Noninvasive blood glucose assay by near-infrared diffuse reflectance spectroscopy of the human inner lip," Appl. Spectrosc. 47(7), 875-881 (1993).

3. K. Maruo et al., "Noninvasive blood glucose assay using a newly developed near-infrared system," Sel.
Topics Quantum Electron. IEEE J. 9(2), 322-330 (2003).

4. E.-J. Suh, Y.-A. Woo, H.-J. Kim, "Determination of water content in skin by using a FT near infrared spectrometer," Arch. Pharm. Res. 28(4), 458-462 (2005).

5. D. Sia, "Design of a near-infrared device for the study of glucose concentration measurements." EE 4BI6 Electrical Engineering Biomedical Capstones, (2010).

6. K. Youcef-Toumi, V. A. Saptari, "Noninvasive blood glucose analysis using near-infrared absorption spectroscopy," The Home Automation and Healthcare Consortium, 1999, p. 2-3.

7. O. Abdallah et al., "Design of a compact multisensor system for noninvasive glucose monitoring using optical spectroscopy," ICEBEA 2012, 2012.

8. K. Lawrence et al., "Evaluation of LED and tungstenhalogen lighting for fecal contaminant detection." Appl. Eng. Agric. 23(6), 811-818 (2007).

9. L. Yuan, "White-light interferometric fiber-optic strain sensor from three-peak-wavelength broadband LED source," Appl. Optics 36(25), 6246-6250 (1997).

10. D. Barolet, "Light emitting diodes (LEDs) in dermatology," in Seminars in Cutaneous Medicine and Surgery, Elsevier, 2008.

11. K. J. Jeon et al., "Comparison between transmittance and reflectance measurements in glucose determination using near-infrared spectroscopy," J. Biomed. Optics 11(1), 014022-014022-7.

12. S. F. Malin et al., "Noninvasive prediction of glucose by near-infrared diffuse reflectance spectroscopy," Clin. Chem. 45(9), 1651-1658 (1999).

13. D. A. Burns, E. W. Ciurczak, Handbook of Nearinfrared Analysis, CRC Press, 2007. 